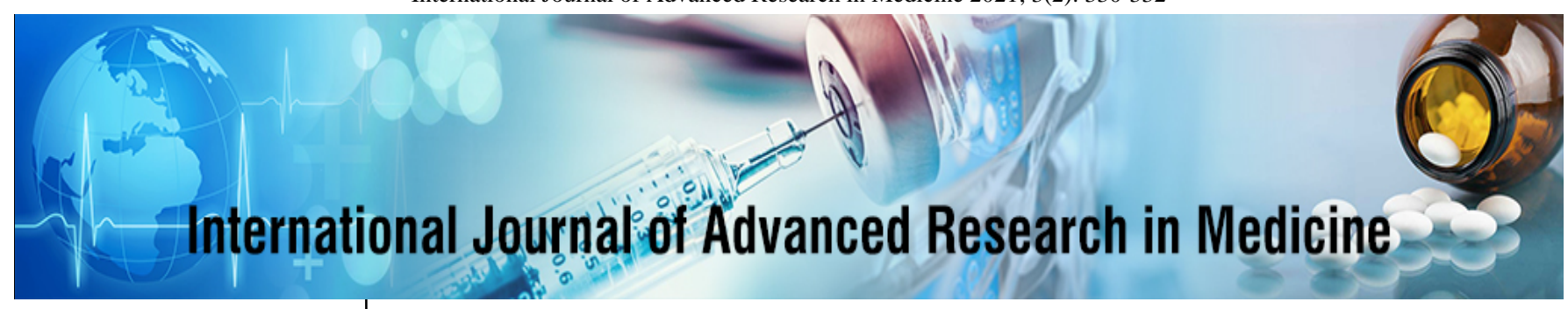

E-ISSN: 2706-9575

P-ISSN: 2706-9567

IJARM 2021; 3(2): 330-332

Received: 02-05-2021

Accepted: 15-06-2021

Dr. Vemulapally Lavanya Final year postgraduate, Department of General medicine, Kamineni Academy of Medical Sciences and Research Centre, L.B Nagar, Hyderabad, Telangana, India

Nimmagadda Venkata Sri Rithish

House Surgeon, Kamineni Academy of Medical Sciences and Research Centre, L.B Nagar, Hyderabad, Telangana, India

Dr. Samaleti Bharath Kumar Final year postgraduate, Department of General medicine, Kamineni Academy of Medical Sciences and Research Centre, L.B Nagar, Hyderabad, Telangana, India

Dr. Miryala Swamy Professor \& HOD, Department of General medicine, Kamineni Academy of Medical Sciences and Research Centre, L.B Nagar, Hyderabad, Telangana, India

Corresponding Author: Dr. Vemulapally Lavanya Final year postgraduate, Department of General medicine, Kamineni Academy of Medical Sciences and Research Centre, L.B Nagar, Hyderabad, Telangana, India

\section{A fungal epidemic in viral pandemic}

\author{
Dr. Vemulapally Lavanya, Nimmagadda Venkata Sri Rithish, Dr. \\ Samaleti Bharath Kumar and Dr. Miryala Swamy
}

\section{DOI: $\underline{\text { https://doi.org/10.22271/27069567.2021.v3.i2f.263 }}$}

\begin{abstract}
Introduction: Covid 19 pandemic continues to be a major health problem worldwide. Despite being more than one year into pandemic, the treatment remains controversial and systemic steroids have shown some benefit. On the flip side rampant use of glucocorticoids has potentially resulted in secondary bacterial and fungal infection. In the early part of pandemic less than $1 \%$ cases were fungal. In 2nd wave of pandemic it has been increasing as an opportunistic infection in covid 19 recovered patients. Mucormycosis infection typically effects immunocompromised individuals, uncontrolled diabetics mellitus, acquired immunodeficiency syndrome, malignancy. Here i report association of covid19, diabetes mellitus, steroid risk in mucormycosis.

Material and methods: A retrospective observational study conducted at tertiary centre, involving all patients with mucormycosis during may 2021 to july 2021.Twenty patients diagnosed with mucormycosis were included in this study. The demographic features, predisposing conditions, clinical features, radiological manifestation and patients outcome were collected from medical records.

Results: Predisposing factors were identified in 20 patients- 5 patients are female, 15 patient are male with diabetics in 17 patients with history of covid in 14 patients, 3 patients non covid. History of covid in 17 patients, 3 non covid patients. Steroids usage was seen in 15 patients of which 14 patients are diabetic and 1 non-diabetic, 11 patients had uncontrolled diabetics.1 patient had ckd. All patients tissue samples collected and submitted for histopathological examination showed fungal hyphae. All our patients MRI was done and extent of involvement of sinuses involvement in 12 patients, rhino orbital in 6 patients, rhino orbital cerebral in 2 patients. 18 patients were recovered and 2 patients were expired. Discussion: Diabetes mellitus directly contributes to mucormycosis risk by hyperglycation of iron sequestering upregulation of cell receptors, defects in phagocytic function and expression of receptors that mediate host cell invasion. Covid 19 has been shown to affect the beta cells of pancreas, causing metabolic derangement leading to diabetes mellitus. Overuse, misuse and rampant use of steroids in covid 19 patients have a increased chance of mucormycosis. Diabetes mellitus when combined with SARS cov 2 virus and steroid therapy, appears to be a vicious cycle of hyperglycemia and immunosuppression, which leads to mucormycosis

Conclusion: The second wave of covid 19 in India has led to more deaths than first wave and in just few weeks.b.1.617.2 delta variant became dominant strain, more infectious and somehow promoting entry of mucorales in to patients. It was declared as epidemic on 21 st may 2021 as there were huge rise in cases. Strict glycemic control, proper sanitization and covid preventive measures should be strictly followed to prevent this deadly fungal infection.
\end{abstract}

Keywords: Covid-19, Mucormycosis, Diabetes Mellitus, Thrombosis, Fungal Strain, Posaconazole

\section{Introduction}

Covid 19 pandemic continues to be a major health problem worldwide. Despite being more than one year into pandemic, the treatment remains controversial and systemic steroids have shown some benefit. On the flip side rampant use of glucocorticoids has potentially resulted in secondary bacterial and fungal infection. In the early part of pandemic less than $1 \%$ cases are fungal. In 2 ND wave of pandemic, it has been increasing as an opportunistic infection in covid 19 recovered patients. Mucor mycosis infection typically effects immunocompromised individuals, uncontrolled diabetics mellitus, acquired immunodeficiency syndrome, malignancy ${ }^{[2]}$. Here I report association of covid-19, diabetes mellitus, steroid risk in Mucormycosis.

\section{Material and Methods}

A retrospective observational study conducted at tertiary Centre, involving all patients with Mucormycosis during May 2021 to July 2021.Twenty patients diagnosed with Mucor 
mycosis were included in this study. The demographic features, predisposing conditions, clinical features, radiological manifestation, and patient's outcome were collected from medical records. Affected individuals present with acute sinusitis, fever, headache, nasal congestion, nasal discharge, nasal ulceration, hemiplegia, stroke, stroke, altered mental function, vision loss, ophthalmoplegia ${ }^{[6]}$. In case of rhinoorbitocerebral Mucormycosis bony destruction and invasion of soft tissues of the orbit or intracranial spread were confirmed on MRI imaging, as it is imaging of choice [7]. Tissue sections were reviewed with KO Hstain, diagnosis was made when biopsy revealed characteristic wide, thick walled, ribbon like aseptate hyphal elements that branch at right angles ${ }^{[1]}$.

\section{Results}

Predisposing factors were identified in 20 patients, with diabetics in 17 patients with history of covid in 14 patients, 3 patients non covid. History of covid in 17 patients, 3 non covid patients. Steroid usage was seen in 15 patients of which 14 patients are diabetic and 1 non-diabetic. 1 patient had CKD. All patients tissue samples collected and submitted for histopathological examination showed fungal hyphae. All our patients MRI was done and extent of involvement of sinuses involvement in 12 patients, rhino orbital in 6 patients, rhino orbital cerebral in 2 patients. 18 patients were recovered, and 2 patients were expired.

Table 1: Age distribution of subjects

\begin{tabular}{|c|c|c|c|}
\hline \multicolumn{2}{|c|}{} & Count & \% \\
\hline \multirow{4}{*}{ Age } & $<40$ years & 4 & $20.0 \%$ \\
\cline { 2 - 4 } & 41 to 50 years & 10 & $50.0 \%$ \\
\cline { 2 - 4 } & 51 to 60 years & 4 & $20.0 \%$ \\
\cline { 2 - 4 } & $>60$ years & 2 & $10.0 \%$ \\
\cline { 2 - 4 } & Total & 20 & $100.0 \%$ \\
\hline
\end{tabular}

Table 2: Gender distribution

\begin{tabular}{|c|c|c|c|}
\hline \multicolumn{2}{|c|}{} & Count & \% \\
\hline \multirow{2}{*}{ Sex } & Female & 5 & $25.0 \%$ \\
\cline { 2 - 4 } & Male & 15 & $75.0 \%$ \\
\hline
\end{tabular}

Table 3: Diagnosis distribution

\begin{tabular}{|c|c|c|c|}
\hline \multicolumn{1}{|c|}{} & Count & \% \\
\hline \multirow{4}{*}{ Diagnosis } & B/L Ethmoid and Sphenoidal Sinusitis & 1 & $5.0 \%$ \\
\cline { 2 - 4 } & Invasive Fungal Sinusitis & 9 & $45.0 \%$ \\
\cline { 2 - 4 } & Left Maxillary Mucor mycosis & 1 & $5.0 \%$ \\
\cline { 2 - 4 } & Mucor Of Left Sinus & 4 & $20.0 \%$ \\
\cline { 2 - 4 } & Mucor Of Right Maxillary Sinusitis & 1 & $5.0 \%$ \\
\cline { 2 - 4 } & Mucor Of Right Sinus & 1 & $5.0 \%$ \\
\cline { 2 - 4 } & Mucor Of Rt Paranasal Sinuses & 1 & $5.0 \%$ \\
\cline { 2 - 4 } & Rhiniorbitocerebral Mucor mycosis & 2 & $5.0 \%$ \\
\cline { 2 - 4 } & Righty Maxillary Mucor mycosis & 1 & $5.0 \%$ \\
\hline
\end{tabular}

Table 4: Comorbidities distribution

\begin{tabular}{|c|c|c|c|}
\hline \multicolumn{2}{|c|}{} & Count & \% \\
\hline \multirow{2}{*}{ DM } & No & 4 & $20.0 \%$ \\
\cline { 2 - 4 } & Yes & 16 & $80.0 \%$ \\
\hline \multirow{2}{*}{ H/O Covid } & No & 3 & $15.0 \%$ \\
\cline { 2 - 4 } & Yes & 17 & $85.0 \%$ \\
\hline \multirow{2}{*}{ Steroids } & No & 5 & $25.0 \%$ \\
\cline { 2 - 4 } & Yes & 15 & $75.0 \%$ \\
\hline
\end{tabular}

Table 5: HbA1c distribution

\begin{tabular}{|c|c|c|c|}
\hline \multicolumn{2}{|c|}{} & Count & \% \\
\hline \multirow{3}{*}{ HbA1c } & $<6.5 \%$ & 4 & $20.0 \%$ \\
\cline { 2 - 4 } & 6.5 to $8 \%$ & 5 & $25.0 \%$ \\
\cline { 2 - 4 } & 8 to 10 gm\% & 5 & $25.0 \%$ \\
\cline { 2 - 4 } & $>10$ gm\% & 6 & $30.0 \%$ \\
\hline
\end{tabular}

\section{Discussion}

Diabetes mellitus directly contributes to Mucormycosis risk by hyperglycation of iron sequestering proteins, disrupting normal iron sequestration, up regulation of mammalian cell receptor grp78 that bind to Mucorales, enabling tissue penetration, induction of defects in phagocytic function, enhanced expression of cot8, a Mucorales specific protein that mediates host cell invasion by binding to grp78.

Covid 19 has been shown to affect the beta cells of pancreas, causing metabolic derangements leading to diabetes mellitus. Grp 78 is a class of heat shock protein released from endoplasmic reticulum of endothelial cells in response to stress. By up regulating of grp78 by virus for its own entry into tissues, it facilitates the fungal endocytosis through same mechanism, it also enhances its pathogenicity and virulence. Mucormycosis is the extensive angioinvasion with resultant vessel thrombosis and tissue necrosis.

Overuse, misuse, and rampant use of steroids in covid 19 patients have increased chance of Mucormycosis. Most common adverse effect of steroids are drug induced hyperglycemia, which aggravate hyperglycemia in known diabetic patients.

Diabetes mellitus when combined with SARS COV-2 virus and steroid therapy, appears to be a vicious cycle of hyperglycemia and immunosuppression, which leads to Mucormycosis ${ }^{[4]}$.

In case of severe COVID 19 infections associated with fungal coinfection, cell count revealed that there was a progressive increase in white blood cell count and neutrophil while lymphocytes progressively decreased. SARS COV2 affects CD4 and CD8 T cells, which are highly involved in pathological progress of COVID, in severe infection there is decreased lymphocytes and T cells. Mucorales specific T cells produce cytokines such as IL4, IL10, IL17 AND IFN-gamma that damage fungal hyphae ${ }^{\text {[3] }}$. Lymphopenia could increase risk of developing invasive Mucormycosis, while improvement of production of Mucorales specific $\mathrm{T}$ cells, it has control over invasive fungal infection.

The second wave of covid 19 in India has led to more deaths than first wave and in just few weeks b.1.617.2 delta variant became dominant strain, more infectious and somehow it is promoting entry of Mucoralesinto patients ${ }^{[9]}$. It was declared as epidemic on 21st May 2021 as there was huge rise in cases ${ }^{[10]}$. Most mucor molds are incapable of infecting humans because they don't grow at body temperature, however some thermotolerant species are isolated from covid 19 associated mucormycosis.it is potentially fatal infection which arises from invasion blood vessels by fungal elements leading to mycotic thrombosis, ischemia, infarction and ultimately necrosis of affective host tissue. Prevalence was 0.14 per 1000 , it was 80 times higher than developed countries, as India is highest for diabetes mellitus cases. It is also combined with other factors like unhygienic conditions, prolonged hospitalization, use of ventilators, leaky humidifiers in oxygen cylinders, they create an ideal environment for contracting. 


\section{Conclusion}

Mucormycosis. Steroid's usage should be only in moderate and severe cases as per doses approved, strict glycemic control and proper sanitization, preventive measures for covid virus should be strictly followed to prevent this deadly fungal infection which has high mortality.

\section{Acknowledgment}

The author is thankful to Department of General Medicine for providing all the facilities to carry out this work.

\section{Conflict of Interest \\ None}

\section{Funding Support}

Nil

\section{References}

1. Mehta S, Pandey A. Rhino-Orbital Mucormycosis Associated With COVID-19. Cureus 2020;12(9):e10726. Published 2020 Sep 30. doi:10.7759/cureus.10726

2. John TM, Jacob CN, Kontoyiannis DP. When Uncontrolled Diabetes Mellitus and Severe COVID-19 Converge: The Perfect Storm for Mucormycosis. J Fungi (Basel) 2021;7(4):298. Doi: 10.3390/jof7040298. PMID: 33920755; PMCID: PMC8071133

3. Revannavar SM, PSS, Samaga L et al. COVID-19 triggering mucormycosis in a susceptible patient: a new phenomenon in the developing world BMJ Case Reports CP 2021;14:e241663.

4. Sharma S, Grover M, Bhargava S, Samdani S, Kataria T. Post coronavirus disease mucormycosis: A deadly addition to the pandemic spectrum. The Journal of Laryngology \& Otology 2021;135(5):442-447. Doi: $10.1017 /$ S0022215121000992.

5. Kondapavuluri Sushen Kumar et al. Spectrum of MR Imaging Findings of Sinonasal Mucormycosis in Post COVID-19 Patients. The British Journal of Radiology 2021, 20210648.

6. Grach Stephanie L et al. Rhinorbitocerebral Mucormycosis in Uncontrolled Diabetes. Mayo Clinic Proceedings 2021;96(10):2651-2652.

7. Kaushik Kavya S et al. Spectrum of Intracranial Complications of Rhino-orbito-cerebral Mucormycosis - Resurgence in the Era of COVID-19 Pandemic: a Pictorial Essay. Emergency Radiology 2021.

8. Pradhan Pradeep et al. Predisposing Factors of Rhinoorbital-cerebral Mucormycosis in Patients with COVID 19 Infection. Indian Journal of Otolaryngology and Head and Neck Surgery: Official Publication of the Association of Otolaryngologists of India 2021, 1-7.

9. Ravindra Khaiwal, Ajit Ahlawat. Five Probable Factors Responsible for COVID-associated Mucormycosis Outbreak in India. International Journal of Infectious Diseases: IJID: Official Publication of the International Society for Infectious Diseases 2021.

10. Arora Shitij et al. Online Registry of COVID-19Associated Mucormycosis Cases, India, 2021. Emerging Infectious Diseases 2021, 27(11). 